\title{
Asymptomatic Breast Carcinoma Representing With Gastric Metastasis: A Case Report and Review of the Literature
}

\author{
Faruk Karateke ${ }^{\mathrm{a}, \mathrm{d}}$, .Pelin Demirturk ${ }^{\mathrm{b}}$, Ebru Menekse ${ }^{\mathrm{a}}$, Koray Das ${ }^{\mathrm{a}}$, \\ Sefa Ozyazicia, Ilhan Balia, Mehmet Ozdogan ${ }^{\mathrm{c}}$
}

\begin{abstract}
The stomach is an unusual site of metastasis from breast carcinoma, and this has rarely been reported in the literature. We report the case of a 50-year-old woman diagnosed with metastatic gastric cancer who was subsequently confirmed to be consistent with metastasis from the invasive lobular carcinoma of the breast primary.
\end{abstract}

Keywords: Breast; Cancer; Stomach; Metastasis

\section{Introduction}

The incidence of breast cancer metastasis to the stomach has been reported at 2-18\% and usually occurs many years after the inital diagnosis of primary breast cancer $[1,2]$. It may be difficult to differentiate a primary gastric cancer from secondary based on clinical, endoscopic, radiological and histopathological findings [1, 3]. However, the differentiation is very important in order to manage these patients to avoid over-treatment. Most series report a greater tendency for lobular carcinoma to metastasize to the gastrointestinal tract $[4,5]$. In this report, we described a case of breast cancer metastasis to the stomach, which was initially diagnosed and treated as primary gastric cancer, and subsequently diagnosed as a metastatic spread of breast origin after pathological evaluation.

\footnotetext{
Manuscript accepted for publication January 22, 2013

${ }^{a}$ Numune Training and Research Hospital, Department of General Surgery. Adana, Turkey

${ }^{b}$ Numune Training and Research Hospital, Department of Pathology. Adana, Turkey

${ }^{c}$ Medline Hospital, Department of General Surgery. Adana, Turkey

${ }^{\mathrm{d} C}$ Corresponding author: Faruk Karateke, Numune Training and Research Hospital, Department of General Surgery. 01170.Adana, Turkey. Email: karatekefaruk@hotmail.com
}

doi: http://dx.doi.org/10.4021/jmc1067w

\section{Case Report}

A fifty-year-old postmenopausal woman was admitted to our clinic with the complaints of weight loss and epigastric pain. Physical examination revealed no abnormality except for cachexia. Laboratory tests including hemogram, biochemical parameters and tumor markers were unremarkable. She was initally investigated with an upper gastrointestinal endoscopy (GE). GE demonstrated diffuse hyperemic nodules in the mucosa of the greater curvature and the corpus posterior wall. Hematoxylin and eosin $(\mathrm{H} \& \mathrm{E})$ staining of the biopsy specimens revealed diffuse carcinoma with signet-ring type. Computed tomography (CT) showed thickened gastric wall (Fig. 1). Based on these findings total gastrectomy with Roux-en-Y esophagojejunostomy reconstrcuction was performed. Histopathology after surgery revealed involvement of the stomach by a malignant neoplasm composed of diffuse infiltration of the muscularis propria and serosa (Fig. 2, 3). Immunohistochemistry showed positive staining for CK 7, GCDFP-15 (gross cystic disease fluid protein-15), ER and PR, where as negative for CK 20.

Due to the suspicion of breast origin, mammography and breast USG was performed. Mammography revealed microcalcificiations in the upper outer quadrant with no mass in left breast of patient while USG showed a solid nodule mea-

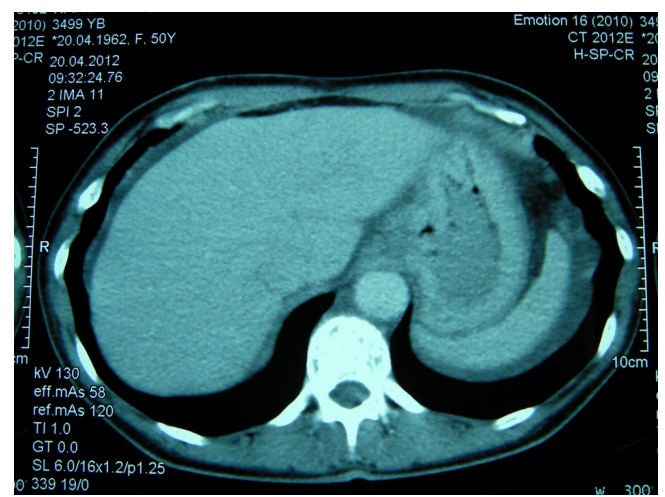

Figure 1. CT abdomen of the patient demonstrating thickening in gastric wall. 


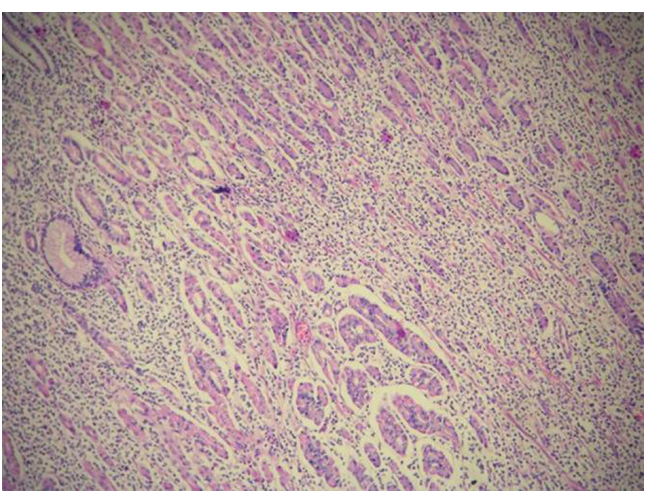

Figure 2. Diffuse infiltrating tumor between regular structure of the gastric mucosal glands. H\&E $\times 100$.

suring $18 \mathrm{~mm} \times 13 \mathrm{~mm}$. The contralateral breast was normal and both axillary was impalpable with nodes. The mass was non-palpable so a USG-guided tru-cut biopsy of the lesion was done, which revealed infiltrating lobular carcinoma. Finally, the patient was evaluated as metastatic breast cancer masquerading as primary gastric cancer and therefore simple mastectomy was performed and the patient was referred to medical oncology for adjuvant therapy.

\section{Discussion}

It has been known that gastric metastasis from breast cancer are rare entities that are usually found after the diagnosis or treatment of the primary tumor. It is very rare to determinate such kind of metastasis in the absence of known primary breast cancer. In this report we presented a case of gastric metastasis from asymptomatic breast cancer admitted to our clinic with the complaints of weight loss and epigastric pain.

Primary and metastatic cancers of the stomach usually presents similiarly with non-specific symptoms of anorexia, vomiting, epigastric pain and/or haemorrhage [2]. These non-specific symptomatolgoy can usually delay the diagnosis as seen in our case. Endoscopic, radiological and histological assessments are usually unhelpful to discriminate between primary gastric cancer and breast cancer metastasis to the stomach. Since metastatic gastric infiltration is frequently limited to the submucosa and seromuscular layer, endoscopic evaluation may be normal in $50 \%$ of cases or only show discrete mucosal abnormalities such as nodular and polypoid or ulcerated lesions indistinguishable from other tumors or benign disease [4]. In their review of the literature from 1943 to June 2012, Massimo et al found that 125 patients has been reported with gastric metastasis from breast cancer including 57 presenting with linitis plastica, 27 with obstruction/stenosis, 12 with ulcer erosion/bleeding, 11 with dyspepsia, 10 with pain as the main symptom, 2 with polypoid lesions, 2 with perforation, and in 4 cases with no

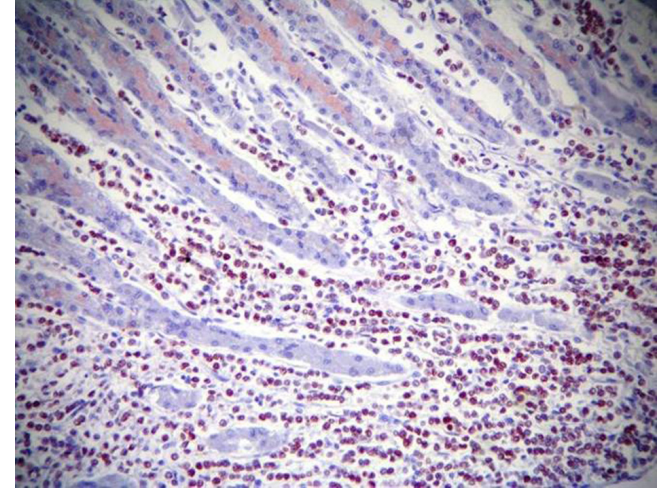

Figure 3. ER positivity by immunohistochemistry. H\&E $\times$ 200.

symptoms in presentation [5].

Imaging findings on CT scan or barium studies include encasement of the whole stomach as seen in linitis plastica, multiple lesions of the stomach or extrinsic lesions of the gastric wall. Deep biopsies are needed to obtain representative material and the histological features should be compared with those of the primary breast tumor in order to confirm the diagnosis. Occasionally, lobular breast carcinoma may produce a signet ring morphology which can be confused with a primary gastric adenocarcinoma as in our case $[6,7]$. The large number of signet-ring cells combined with a gastric mucosal spreading pattern can compromise the diagnosis, and metastatic disease to the stomach can be almost indistinguishable from primary gastric linitis plastica [7]. Madeya et al reported that $73 \%$ of patients with gastric metastases had diffuse intramural infiltration imitating linitis plastica [8].

Further immunohistochemical studies may be the only reliable method to differentiate between metastatic and primary gastric carcinoma. Metastatic breast carcinoma is usually positive for CK7, GCDFP-15, carcinoembryonic antigen (CEA), ER and PR, and negative for CK20 [7]. Although ER and PR positivity in gastric biopsies suspects breast cancer metastasis to the stomach, it is has been estimated that ER and PR positivity with weak to moderate staining intensity in $32 \%$ and $12 \%$, respectively, of patients with primary gastric cancer [7]. ER $\alpha$ can be used to differantiate gastric metastasis from breast cancer as van Velthuysen et al reported that no primary gastric tumor expressed ER $\alpha$. They also observed that the absence of E-cadherin staining was significantly related to metastatic breast carcinoma [9]. Positive staining with GCDFP-15 has been found to be a sensitive (55-76\%) and specific (95-100\%) marker for correctly identifying a malignant lesion as metastatic breast carcinoma $[3,7,10]$. The positivity for CK7 and GCDFP-15 including hormone receptor expression of ER $\alpha$.and PR, and in addition the negativity for CK20 were of great value in accurated diagnosing in the presented case. 
Most series report a higher tendency for lobular carcinoma to metastasize to the gastrointestinal tract, the gynecological organs and the peritoneum while ductal carcinoma most frequently relapses in the liver, the bones, the lungs and the brain $[4,5,7]$.The reason for this mode of spread has not been clearly identified. Taal et al [6] reported that $83 \%$ of patients with gastric involvement had lobular breast cancer as a primary histological subtype as described in our case.

Reports on treatment of gastric metastasis of breast cancer has varied in literature. Breast cancer metastasis to the stomach represents evidence of systemic disease and the need for systemic therapy $[6,11]$. Surgery is usually required to palliate symptoms in the case of obstruction, bleeding and perforation $[1,6,11]$. McLemore et al reported that surgical intervention did not have a significant effect on survival [11]. However, Pectaides et al stated that patients with metastasis only to the gastrointestinal tract who underwent palliative surgical resection tended to have an increased median survival [7].The overall prognosis at a progressed stage from most of the reported case series is rather poor, with a predicted median survival of only $24-36$ months from the time of diagnosis $[5,12]$.

Contrary to the most previous reports, the patient in this report were initially diagnosed with an apparent primary gastric cancer and therofore this diagnosis was revised to metastatic spread of breast cancer, but only after surgery had been performed.

\section{Conlusion}

We remind that metastases to stomach may rarely be the first manifestation of breast cancer metastases. Surgeons should be awake of patients affected by breast cancer who complained of gastrointestinal symptoms. Detailed diagnostic procedures could be required to obtain a conclusive histopathological diagnosis allowing a satisfactory treatment for the patients.

\section{Conflict of Interest}

There is no conflict of interest to declare.

\section{References}

1. Schwarz RE, Klimstra DS, Turnbull AD. Metastatic breast cancer masquerading as gastrointestinal primary. Am J Gastroenterol. 1998;93(1):111-114.

2. Taal BG, den Hartog Jager FC, Steinmetz R, Peterse $\mathrm{H}$. The spectrum of gastrointestinal metastases of breast carcinoma: I. Stomach. Gastrointest Endosc. 1992;38(2):130-135.

3. Yim H, Jin YM, Shim C, Park HB. Gastric metastasis of mammary signet ring cell carcinoma--a differential diagnosis with primary gastric signet ring cell carcinoma. J Korean Med Sci. 1997;12(3):256-261.

4. Borst MJ, Ingold JA. Metastatic patterns of invasive lobular versus invasive ductal carcinoma of the breast. Surgery. 1993;114(4):637-641; discussion 641-632.

5. Ambroggi M, Stroppa EM, Mordenti P, Biasini C, Zangrandi A, Michieletti E, Belloni E, et al. Metastatic breast cancer to the gastrointestinal tract: report of five cases and review of the literature. Int J Breast Cancer. 2012;2012:439023.

6. Taal BG, Peterse H, Boot H. Clinical presentation, endoscopic features, and treatment of gastric metastases from breast carcinoma. Cancer. 2000;89(11):2214-2221.

7. Pectasides D, Psyrri A, Pliarchopoulou K, Floros T, Papaxoinis G, Skondra M, Papatsibas G, et al. Gastric metastases originating from breast cancer: report of 8 cases and review of the literature. Anticancer Res. 2009;29(11):4759-4763.

8. Madeya S, Borsch G. Gastrointestinal metastases of breast carcinoma. Gastrointest Endosc. 1993;39(1):103-104.

9. van Velthuysen ML, Taal BG, van der Hoeven JJ, Peterse JL. Expression of oestrogen receptor and loss of E-cadherin are diagnostic for gastric metastasis of breast carcinoma. Histopathology. 2005;46(2):153-157.

10. Honma N, Takubo K, Arai T, Younes M, Kasumi F, Akiyama F, Sakamoto G. Comparative study of monoclonal antibody B72.3 and gross cystic disease fluid protein-15 as markers of apocrine carcinoma of the breast. APMIS. 2006;114(10):712-719.

11. McLemore EC, Pockaj BA, Reynolds C, Gray RJ, Hernandez JL, Grant CS, Donohue JH. Breast cancer: presentation and intervention in women with gastrointestinal metastasis and carcinomatosis. Ann Surg Oncol. 2005;12(11):886-894.

12. Greenberg PA, Hortobagyi GN, Smith TL, Ziegler LD, Frye DK, Buzdar AU. Long-term follow-up of patients with complete remission following combination chemotherapy for metastatic breast cancer. J Clin Oncol. 1996;14(8):2197-2205. 